\title{
Is newer better? Not always
}

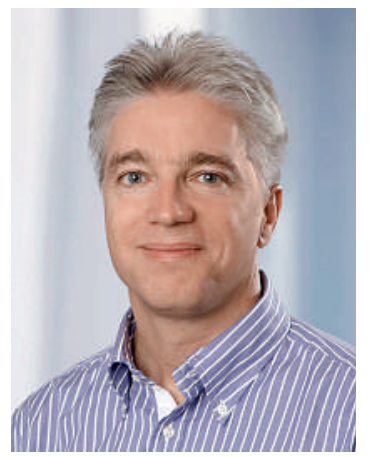

En juin dernier, a paru dans le New York Times un éditorial intitulé «Is newer better? Not always» consacré aux nouvelles technologies et à leurs apports [1]. L'évaluation des technologies de la santé ou Health Technology Assessements (HTA) est un sujet d'une brûlante actualité en politique de la santé. Elle donne lieu à la création de groupes de travail, à l'organisation de symposiums et de tables rondes avec le public. Mais que recouvre ce terme exactement? Pour reprendre un fait bien connu mais que l'on a parfois tendance à oublier, les progrès technologiques, et notamment en médecine, sont de plus en plus écartelés entre le techniquement possible et le financièrement supportable par la collectivité. L'utilité marginale de nombreuses innovations technologiques diminuant continuellement, un débat s'impose autour de ce que nous voulons et surtout, de ce que nous pouvons assumer au titre de l'assurance obligatoire et solidaire. Un certain nombre de solutions avancées, comme celle entendue lors d'un récent symposium de SwissDRG SA, de n'autoriser le recours à des solutions novatrices que par le biais des assurances complémentaires (sic!), méritent un débat approfondi.

La FMH a pris une part active aux initiatives HTA et donc à l'ensemble du débat. Conjointement avec la Conférence suisse des directeurs sanitaires cantonaux et l'Académie suisse des sciences médicales (ASSM), elle est membre du Swiss Medical Board (www.medical-board.ch), une institution qui a déjà réalisé plusieurs études HTA, et siège ainsi

\section{La médecine est de plus en plus écartelée entre le techniquement possible et le financièrement supportable par la collectivité.}

dans un organe stratégique de ce domaine. Nous nous engageons également au sein de SwissHTA en collaboration avec santésuisse, interpharma et l'ASSM en vue de créer un large consensus sur la question et d'élaborer un document exhaustif, à caractère scientifique, qui doit définir ce que les HTA pourraient et devraient être à l'avenir en Suisse.

Les revendications que nous avons formulées au nom du corps médical suisse tournent autour des trois axes suivants: 1. Les processus HTA en Suisse doivent être transparents et compréhensibles. Il convient donc d'impliquer de manière appropriée les experts concernés.
2. Les HTA ne doivent pas être des freins à l'innovation mais au contraire permettre de distinguer entre véritables et pseudo-innovations. Il s'agit d'éviter des processus trop longs et de maintenir la capacité innovatrice de la Suisse et son attractivité dans ce domaine.

3. Les HTA sont un outil qui doit permettre d'étayer un certain nombre de dossiers de politique de la santé, comme la mise en œuvre des critères EAE (efficacité, adéquation, économicité) ou encore les questions de rationnement. Il s'agit en particulier de mettre en évidence les manques en matière de recherche ou d'identifier le degré d'incertitude en lien avec certaines argumentations.

En médecine, le progrès technologique peut être spectaculaire: je venais d'avoir dix ans lorsque j'ai subi une opération

\section{Le corps médical doit s'impliquer dans les procédures d'évaluation des nouveaux processus et des nouvelles technologies en médecine.}

à cœur ouvert de cinq heures avec une hospitalisation de deux semaines et une interdiction d'exercer une quelconque activité sportive pendant une année. Dix ans plus tard, un diagnostic similaire a été établi chez un sportif helvétique de haut niveau quelques mois avant les Jeux olympiques. Une intervention par cathéter ne l'a pas empêché de remporter un titre olympique.

C'est le genre d'innovation dont nous rêvons pour la médecine. Mais de telles avancées ne sont pas quotidiennes. Bien au contraire, aujourd'hui nous sommes confrontés à une affluence de nouveautés dont l'utilité peut paraître très limitée. C'est la raison pour laquelle le corps médical doit s'impliquer activement dans le processus HTA. Grâce à notre expertise, nous pouvons faire valoir notre point de vue dans l'évaluation critique des nouveaux processus et des nouvelles technologies. Une démarche où le bon sens a tout autant sa place que le savoir: new can be better, but not necessarily always!

Dr Daniel Herren MHA

Membre du Comité central de la FMH,

Responsable du domaine Données, démographie et qualité (DDQ)

1 http://www.nytimes.com/2010/09/12/opinion/12sun1.html ?scp=3\&sq=is+new+always+better\%3F\&st=nyt 